\title{
GUT HEALTH AND INTEGRITY IN ATLANTIC SALMON FED PLANT PROTEIN DIETS

\author{
Elżbieta Król $^{1}$, Alex Douglas ${ }^{1}$, Christopher J Secombes ${ }^{1}$ and Samuel A. M. Martin ${ }^{1 \S}$
}

${ }^{I}$ Institute of Biological and Environmental Sciences, University of Aberdeen, Aberdeen AB24 2TZ, UK

\begin{abstract}
Modern feeds for aquaculture have increasing amounts of plant proteins to replace wild sourced marine ingredients. However, plant materials often contain antinutritional factors that can lead to gut inflammation (enteritis) and compromise fish health. Thus, each new plant material has to be evaluated for its potential health risks, in a dose-dependent manner and in the context of potential interactions with other feed ingredients. A feeding trial on Atlantic salmon parr was carried out using varying levels ( $0-45 \%)$ of bean protein concentrate (BPC, produced from faba beans Vicia faba) and soya protein concentrate (SPC) to determine how the inclusion of BPC affected gut morphology and gene expression. Additionally, soybean meal (SBM) and fish meal (FM) were used as positive and negative controls for enteritis, respectively. Our histological examination of distal gut indicated that the FM diet and diets containing $0-34 \%$ of BPC did not cause enteritis, in contrast to SBM and $45 \%$ BPC that induced gut inflammation. Our transcriptomic analysis revealed that mixed-plant protein diets (containing both SPC and BPC) induced less extensive changes in the gut transcriptome than single-plant protein diets (either $45 \%$ SPC or $45 \%$ BPC), consistent with the fish whole body performance. Importantly, alterations of molecular pathways suggested increased permeability and potential damage to the intestinal integrity. Factors relating to immune function and other metabolic processes were altered in a diet-dependent manner. We suggest that intestinal transcriptomic profiling provides an essential tool for testing the applicability of alternative protein sources for aquaculture feeds and designing the diets with reduced impact of antinutritional factors on fish health. This project was part funded by the UK Technology Strategy Board (TSB Grant 11974-81166) and BBSRC grant (BB/M026604/1).
\end{abstract}

\section{KEYWORDS}

Intestine, inflammation, transcriptomics, nutrition

$\S$ Corresponding author:

Samuel A.M. Martin (phone: +44 (0)1224 272867, email: sam.martin@abdn.ac.uk). 